\title{
AN ERRATUM TO: "A NOTE ON COMMON FIXED POINTS BY ALTERING DISTANCES"
}

\author{
K. JHA, R. P. PANT AND V. PANT
}

\begin{abstract}
The aim of this remark is to provide a correction to an error in the paper of Pant et al. [1].
\end{abstract}

The original paper by Pant et al. [1] contains one mistake. In this remark, we provide a minor correction for that mistake in the proof of Theorem 2.1 of [1]. We start with the following Definition and Theorem (2.1 of [1]).

Definition 1. A control function $\Psi$ is defined as $\Psi: \Re^{+} \rightarrow \Re^{+}$which is continuous at zero, monotonically increasing, $\Psi(2 t) \leq 2 \Psi(t)$ and $\Psi(t)=0$ if, and only if $t=0$. It is noted that this function $\Psi$ need not be sub additive [2].

Theorem 1. ([1]): Let $(A, S)$ and $(B, T)$ be weakly commuting pairs of self mappings of a complete metric space $(X, d)$ and the function $\Psi$ be as in definition (1) satisfying

(i) $A X \subset T X, B X \subset S X$ and

(ii) There exists $h$ in $[0,1)$ such that $\Psi(d(A x, B y)) \leq h M_{\Psi}(x, y)$ for all $x, y$ in $X$.

Suppose that $A$ and $S$ are $\Psi$-compatible and $A$ is continuous. Then $A, B, S$ and $T$ have a unique common fixed point.

The error occurs in line 13 on page 61 to line 5 on page 62 (from above) which claim that $A z=S z=T w=B w$. But the given proof in [1] is valid only when $S$ is assumed to be continuous. This leads to contradiction to our assumption on $A$ in [1]. To overcome this problem, the theorem can be proved along the similar lines as given in the original one with minor changes in accordance with the following steps.

Since $A X \subset T X, A z=T w$ for some $w$ in $X$ and corresponding to each $x_{2 n}$, there exists a $w_{2 n}$ such that $A A x_{2 n}=T w_{2 n}$. Thus we have $A A x_{2 n}=T w_{2 n} \rightarrow T w$ and $S A x_{2 n} \rightarrow T w$. Also, sicne $B X \subset S X$, corresponding to each $w_{2 n}$, there corresponds $u_{2 n}$ such that $B w_{2 n}=S u_{2 n}$. Thus, we have $B w_{2 n}=S u_{2 n} \rightarrow T w$ and $T w_{2 n} \rightarrow T w$.

Now, we claim that $A u_{2 n} \rightarrow T w$ as $n \rightarrow \infty$.

For this,

$$
\begin{aligned}
\Psi\left(d\left(A u_{2 n}, B w_{2 n}\right)\right) \leq & h M_{\Psi}\left(u_{2 n}, w_{2 n}\right) \\
= & h \max \left\{\Psi\left(d\left(S u_{2 n}, T w_{2 n}\right)\right), \Psi\left(d\left(A u_{2 n}, S u_{2 n}\right)\right), \Psi\left(d\left(B w_{2 n}, T w_{2 n}\right)\right),\right. \\
& {\left.\left[\Psi\left(d\left(A u_{2 n}, T w_{2 n}\right)\right)+\Psi\left(d\left(S u_{2 n}, B w_{2 n}\right)\right)\right] / 2\right\} . }
\end{aligned}
$$

Received November 27, 2003.

2000 Mathematics Subject Classification. 47H10. 
Taking $n \rightarrow \infty$, we get $\Psi\left(d\left(A u_{2 n}, T w\right)\right)<h \Psi\left(d\left(A u_{2 n}, T w\right)\right)<\Psi\left(d\left(A u_{2 n}, T w\right)\right)$, a contradiction. Thus we have $A u_{2 n} \rightarrow T w$ as $n \rightarrow \infty$.

Also, we claim that $B w=T w$.

If possible, suppose $B w \neq T w$. Then, as $n \rightarrow \infty$, the inequality

$$
\begin{aligned}
\Psi\left(d\left(A u_{2 n}, B w\right)\right) \leq & h M_{\Psi}\left(u_{2 n}, w\right) \\
=h \max \left\{\Psi\left(d\left(S u_{2 n}, T w\right)\right), \Psi\left(d\left(A u_{2 n}, S u_{2 n}\right)\right), \Psi(d(B w, T w)),\right. & {\left.\left[\Psi\left(d\left(A u_{2 n}, T w\right)\right)+\Psi\left(d\left(S u_{2 n}, B w\right)\right)\right] / 2\right\}, }
\end{aligned}
$$

yields $\Psi(d(T w, B w))<h \Psi(d(T w, B w))<\Psi(d(T w, B w))$, a contradiction.

Hence, we get $T w=B w$. Thus we have $A z=T w=B w$.

Again, since $B X \subset S X$, so there exists $u$ in $X$ such that $B w=S u$; that is, $S u=$ $B w=T w$. Finally, we assert that $A u=S u$.

If $A u \neq S u$. Then by virtue of (ii) of Theorem 1, we get

$$
\begin{aligned}
\Psi(d(A u, S u))= & \Psi(d(A u, B w)) \\
< & h M_{\Psi}(u, w) \\
= & h \max \{\Psi(d(S u, T w)), \Psi(d(A u, S u)), \Psi(d(B w, T w)), \\
& {[\Psi(d(A u, T w))+\Psi(d(S u, B w))] / 2\}, } \\
= & h \Psi(d(A u, S u))<\Psi(d(A u, S u)), \text { a contradiction. }
\end{aligned}
$$

Thus, $A u=S u$ and hence we have $A u=S u=B w=T w$.

Since $A$ and $S$ are weakly commuting, we have by (1.1), $A S u=S A u$ and hence

$$
A A u=A S u=S A u=S S u .
$$

Also, applying the weakly commuting property of $B$ and $T$, we get

$$
B B w=B T w=T B w=T T w .
$$

We now finally show that $A A u=A u$.

Suppose on the contrary that $A A u \neq A u$. Then by (ii), we get

$$
\begin{aligned}
\Psi(d(A u, A A u)) & =\Psi(d(A A u, B w)) \\
& \leq h M_{\Psi}(A u, w)=h \Psi(d(A u, A A u)), \quad(\text { using }(1.2) \text { and }(1.3)),
\end{aligned}
$$

a contradiction. Hence, we must have $A A u=A u$. Therefore, $A u$ is a common fixed point of $A$ and $S$.

The remaining part of Theorem 2.1 in [1] remains unaltered. This result along with [1] provides a complete affirmative answer to the open problem posed by Sastry et al. [2]. 


\section{References}

[1] R. P. Pant, K. Jha and A. B. Lohani, A note on common fixed points by altering distances, Tamkang J. Maths. 34(2003), 59-62.

[2] K. P. R. Sastry, S. V. R. Naidu, G. V. R. Babu and G. A. Naidu, Generalization of common fixed point theorems for weakly commuting mappings by altering distances, Tamkang J. Math. 31(2000), 243-250.

Department of Mathematical Sciences, Kathmandu University, P.O. Box No. 6250, Kathmandu, Nepal.

E-mail: jhaknh@yahoo.co.in

Department of Mathematics, Kumaon University, D. S. B. Campus, Nainital- 263 002, Uttaranchal, India. 